\title{
Changes in the fluidity of model lipid membranes evoked by the organophosphorus insecticide methylbromfenvinfos
}

\author{
Janusz Błasiak* \\ University of Łódź, Department of Molecular Genetics, S. Banacha 12/16, 90-237 Łódź, Poland
}

The ability of many insecticides to bind to the lipids form a basis for considering biological membranes as a primary target of interaction of these agents with living organisms [ 1 - 6]. However, the ability of insecticides to bind to the membrane which, generally, is not correlated with their toxicity, is dependent on many factors, such as temperature, cholesterol content, physical-chemical profiles and fluidity of the membrane, and the nature of the insecticide itself [7 - 12]. The latter two seem to be the main factors determining the ability of insecticides to bind to the membrane [13].

In the present work the fluidity of liposomes made of synthetic phosphatidylcholine has been investigated in the presence of organophosphorus insecticide methylbromfenvinfos (2-bromo-1-(2,4-dichlorophenyl)-vinyl dimethyl phosphate).

Although the results obtained on simple artificial membranes cannot be directly extrapolated to the complex natural ones, the well defined subject of such a study allows one to interpret the results obtained in more precise physical-chemical terms.

Liposomes were formed of distearoylphosphatidylcholine (DSPC) ${ }^{1}$ by shaking, sonication and centrifugation. All fluorescence spectra were measured in a Perkin-Elmer luminescence spectrometer, model LS-50 equipped with a thermostated sample cell holder. Interaction of methylbromfenvinfos with membranes was monitored by fluorescence anisotropy of 1,6-diphenyl-1,3,5-hexatriene (DPH), a probe located at the bilayer center, and by intramolecular excimerization of 1,3-bis-(1pyrene)propane ( $\mathrm{Py}(3) \mathrm{Py})$, a probe located in the outer regions of the bilayer. Steady-state fluorescence anisotropy was measured as described in the accompanying communication [14] perpendicularly to the exciting beam according to $[15,16]$.

All the measurements were corrected for parasite light scaterring [17]. DPH was injected from stock solution into the membrane suspension to give a final lipid:DPH molar ratio of 300 . The excitation was set at $336 \mathrm{~nm}$ and the emission observed at $450 \mathrm{~nm}$.

Intramolecular excimer formation, a phenomenon characteristic of the other fluorescence probe used, $\mathrm{Py}(3) \mathrm{Py}$, is caused by association of an excited and an unexcited aromatic group incorporated in the same molecule and can be described by the excimer to monomer fluorescence quantum yield ratio, $\Phi^{\prime} / \Phi$, which is proportional to the fluorescence intensity ratio, $I^{\prime} / I[18,19]$. The latter ratio for $\mathrm{Py}(3) \mathrm{Py}$ was shown to increase with fluidity in highly viscous media [19 - 21]. The technique is therefore suitable for determination of fluidity of the probe environment in such media as lipid bilayers. Aliquots of the stock solution of Py(3)Py $(0.1 \mathrm{mM}$ in ethanol) were added to the membrane suspension to give a final probe:lipid ratio of 1:900. The excitation was set at $342 \mathrm{~nm}$, emission of monomer, depending on temperature, either at 377 or $378 \mathrm{~nm}$, emission of excimer at $490 \mathrm{~nm}$. After a period of incubation

"Present address: Louisiana State University, Department of Chemistry, 232 Choppin Hall, Baton Rouge, LA 70803-1804, U.S.A.

${ }^{1}$ Abbreviations: DPH, 1,6-diphenyl-1,3,5-hexatriene; DSPC, distearoylphosphatidylcholine; $\mathrm{Py}(3) \mathrm{Py}$, 1,3-bis-(1-pyrene)propane 
with the probes, methylbromfenvinfos was added from stock $(50 \mathrm{mM})$ ethanolic solution to give a final concentration of $50 \mu \mathrm{M}$. The samples were equilibrated for the period of $1 \mathrm{~h}$ $10^{\circ} \mathrm{C}$ above the temperature of the main phase transition.

No changes were observed in fluorescence anisotropy of DPH either in the fluid or solid phase of DSPC vesicles in the presence of 50 $\mathrm{mM}$ methylbromfenvinfos (Fig. 1A). The phase transition profile of the insecticide treated liposomes was somewhat broadened compared to the control. The insecticide evoked a shift in the middle temperature of phase transition $\left(\mathrm{T}_{\mathrm{m}}\right)$ towards the lower temperature.

The presence of the insecticide caused a significant lowering of the ratio $I^{\prime} / I$ in the fluid phase as indicated by the probe Py(3)Py (Fig. 1B). However, there were no changes in the $T_{m}$ of the main transition. In the control vesicles a pretransition was detected, which also was abolished by the insecticide. No changes were observed in the solid phase.

The use of two fluorescent probes distributed in different regions of the bilayer permits to conclude that methylbromfenvinfos perturbs selectively the fluidity of the membrane: a more distinct effect was observed when information came from the highly cooperative region of the membrane structure. Thus, the insecticide binds probably to the membrane in this region. The decrease of $T_{m}$ and broadening of the transition profile support this hypothesis because such phenomena are characteristic of compounds known to be localized in the cooperativity region [22]. Localization of methylbromfenvinfos in this membrane region may help to explain the different effects of the insecticide on the membrane in the fluid phase demonstrated with the use of DPH and Py(3)Py. The insecticide would increase the ordering (solidification) of lipids by increasing their packing density in the region where it is accumulated, but would not affect this ordering measurably in the region where it accumulates in much lower concentration.

Lowering of $\mathrm{T}_{\mathrm{m}}$ of the transition indicated by $\mathrm{DPH}$, and lack of this effect in the case of Py(3)Py allows to conclude that methylbromfenvinfos evokes phase separation.

In addition to the main thermotropic phase transition, $\mathrm{Py}(3) \mathrm{Py}$ indicated also a pretransition in DSPC (Fig. 1B). Since this pretransition occurs in the cooperativity region [23] and is abolished by the presence of $50 \mu \mathrm{M}$ methylbromfenvinfos, thus provides further evidence that the insecticide is located in this region of the membrane.

This study was performed at the Department of Zoology, University of Coimbra, Portugal.

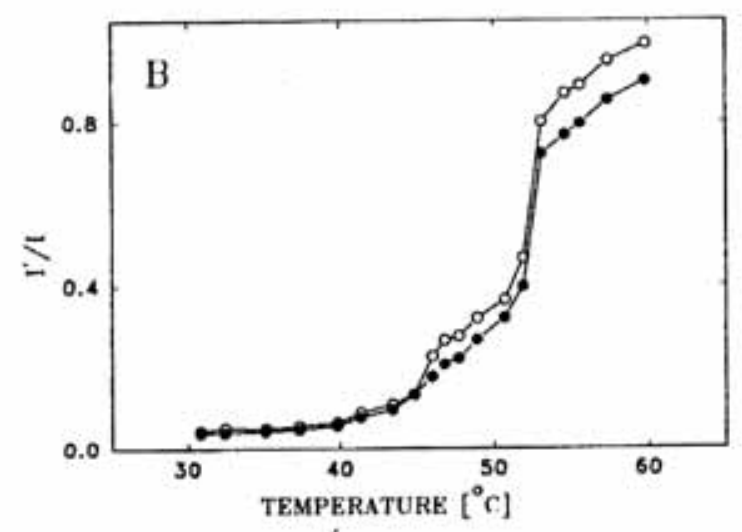

Fig. 1. Fluorescence anisotropy of $D P H(A)$ and intramolecular excimerization of $P y(3) P y(B)$ of distearoylphosphatidylcholine liposomes in the absence $(O)$ and in the presence $(O)$ of $50 \mu \mathrm{M}$ methylbromfenvinfos. Each experimental point is the mean of three measurements. Error bars were omitted to increase the clarity of the picture 
Thanks to Drs V.M.C. Madeira and M.C. Madeira for help in this research.

\section{REFERENCES}

1. Eto, M. (1974) Organophosphorus Pesticides: Organic and Biological Chemistry; pp. 123 - 333, CRC Press, Cleveland.

2. Kuhr, R.J. \& Dorough, H.W. (1976) Carbamate Insecticide: Chemistry, Biochemistry and Toxicology; pp. 41 - 70, CRC Press, Cleveland.

3. Narahashi, T. \& Haas, H.G. (1968) J. Gen. Physiol. 51, 177 - 198.

4. Doherty, J.D. (1979) Pharmacol. Ther. 7, 123 - 151.

5. Metcalf, R.L. (1982) Neurotoxicol. 3, 269 - 284.

6. Corbett, J.R., Wright, K. \& Baillie, A.C. (1984) in Biochemical Mode of Action of Pesticides (Coats, J.R., ed.) pp. 141 - 179, Academic Press, London.

7 Antunes-Madeira, M.C. \& Madeira, V.M.C. (1979) Biochim. Biophys. Acta 550, 384 - 392.

8. Antunes-Madeira, M.C., Carvalho, A.P. \& Madeira, V.M.C. (1980) Pestic. Biochem. Physiol. 14, 161 - 169.

9. Antunes-Madeira, M.C. \& Madeira, V.M.C. (1985) Biochim. Biophys. Acta 820, 165 - 172.

10. Antunes-Madeira, M.C. \& Madeira, V.M.C. (1984) Biochim. Biophys. Acta 778, 49 - 56.

11. Antunes-Madeira, M.C. \& Madeira, V.M.C. (1986) Biochim. Biophys. Acta 861, 159 - 164.

12. Metcalf, R.L. (1971) in Pesticide in the Environment (Whitestevens, R., ed.) vol 1, pp. 1 - 144, Marcel Dekker, New York.

13. Antunes-Madeira, M.C. \& Madeira, V.M.C. (1989) Biochim. Biophys. Acta 982, 161 - 166.

14. Blasiak, J. (1993) Acta Biochim. Polon. 40, 35 - 38.

15. Schinitzky, M. \& Inbar, M. (1974) J. Mol. Biol. 85, $603-615$.

16. Schinitzky, M. \& Barenholtz, Y. (1974) J. Biol. Chem. 249, 2652 - 2657.

17. Kuhry, J.-G., Duportail, G., Bronner, C. \& Laustriat, G. (1985) Biochim. Biophys. Acta 845, 60 $-67$.

18. Zachariasse, K.A., Kuhnle, W. \& Weller, A. (1978) Chem. Phys. Lett. 59, 375 - 380.

19. Zachariasse, K.A., Kuhnle, W. \& Weller, A. (1980) Chem. Phys. Lett. 73, 6 - 11.

20. Melnick, R.J., Haspel, H.C., Goldenberg, M., Greenbaum, L.M. \& Weinstein, S. (1981) Biophys. J. 34, 499 - 515.

21. Zachariasse, K.A. (1978) Chem. Phys. Lett. 57, 429 $-432$.
22. Jain, M.K. \& Wu, N.M. (1977) J. Membr. Biol. 34, $157-201$.

23. Almeida, L.M.,Vaz, W.L.C., Zachariasse, K.A. \& Madeira, V.M.C. (1984) Biochemistry 23, 4714 4720. 